\title{
Charginos and neutralinos in the light of radiative corrections: Sealing the fate of Higgsino dark matter
}

\author{
John Ellis \\ TH Division, CERN, Geneva, Switzerland \\ Toby Falk \\ Department of Physics, University of Wisconsin, Madison, Wisconsin 53706 \\ Gerardo Ganis \\ Max-Planck-Institut für Physik, Munich, Germany \\ Keith A. Olive \\ School of Physics and Astronomy, University of Minnesota, Minneapolis, Minnesota 55455 \\ Michael Schmitt* \\ PPE Division, CERN, Geneva, Switzerland \\ (Received 30 January 1998; published 18 September 1998)
}

\begin{abstract}
We analyze the CERN LEP constraints from searches for charginos $\chi^{ \pm}$and neutralinos $\chi_{i}$, taking into account radiative corrections to the relations between their masses and the underlying Higgs-mixing and gaugino-mass parameters $\mu, m_{1 / 2}$, and the trilinear mass parameter $A_{t}$. While radiative corrections do not alter the excluded domain in $m_{\chi^{ \pm}}$as a function of $m_{\chi^{ \pm}}-m_{\chi}$, its mapping into the $\mu, m_{1 / 2}$ plane is altered. We update our previous lower limits on the mass of gaugino dark matter and on $\tan \beta$, the ratio of Higgs vacuum expectation values, in light of the latest LEP data and these radiative corrections. We also discuss the viability of Higgsino dark matter, incorporating coannihilation effects into the calculation of the Higgsino relic abundance. We find that Higgsino dark matter is viable for only a very limited range of $\mu$ and $m_{1 / 2}$, which will be explored completely by upcoming LEP runs. [S0556-2821(98)03317-7]
\end{abstract}

PACS number(s): 14.80.Ly, 12.60.Jv

\section{INTRODUCTION}

Considerable experimental effort is currently being devoted at the CERN $e^{+} e^{-}$collider LEP and elsewhere to the search for the charginos $\chi^{ \pm}$and neutralinos $\chi_{i} ; i$ $=1, \ldots, 4$ of the minimal supersymmetric extension of the standard model (MSSM) [1-4], and to understanding the constraints these and other particle searches impose on the parameter space of the MSSM [5,6]. One of the generic possibilities for MSSM [7] phenomenology is that supersymmetry breaking is communicated to the observable sparticles via gravitational interactions, in which case the lightest supersymmetric particle is presumably the lightest neutralino $\chi_{1}$ (hereafter $\chi$ ), which would be stable if $R$ parity is conserved. The lightest neutralino is not directly visible to accelerator experiments in this type of $R$-conserving supergravity scenario, and would be a good candidate $[8,9]$ for the cold dark matter advocated by many astrophysicists and theorists of cosmological structure formation [10], since it might well have $0.1 \leqslant \Omega_{\chi} h^{2} \leqslant 0.3$, where $\Omega_{\chi}$ is the relic density of the lightest neutralino $\chi$ in units of the critical density, and $h$ is the present Hubble expansion rate in units of $100 \mathrm{~km} / \mathrm{s} / \mathrm{Mpc}$. Within this framework, which we adopt in this paper, detailed modeling of the relations between different searches is often necessary in order to close certain loopholes in the

\footnotetext{
*Present address: Department of Physics, Harvard University,
} Cambridge, Massachusetts 02138 .
MSSM parameter space, in particular if one wishes to establish robust lower limits on the mass of the lightest neutralino $\chi[11,4]$. Such lower limits on $m_{\chi}$ are of particular relevance to the ongoing direct and indirect nonaccelerator searches for cosmological relic neutralinos [10].

The strength of the lower limit obtained depends $[5,6]$ on various theoretical assumptions, such as the degree of universality among input supersymmetry-breaking parameters, and whether one requires that the relic density of neutralinos lie within the range favored by astrophysical and cosmological considerations [10]. The successive upgrades of the LEP 2 center-of-mass energy [1-3] have enabled this lower limit to be strengthened progressively, and it currently stands at $m_{\chi} \gtrsim 40 \mathrm{GeV}$ if the strongest versions of these theoretical and astrophysical assumptions are imposed [6].

Although radiative corrections to MSSM Higgs scalar masses [12] have been included in the analysis of LEP data [4], and play an important role, radiative corrections to the masses of the charginos and neutralinos have not so far been included. The one-loop corrections to the relations between the physical masses and underlying MSSM parameters such as the gaugino masses $M_{a}: a=1,2,3$ and the Higgs-mixing parameter $\mu$ are by now well known [13-15] and non-negligible. ${ }^{1}$ In particular, they are known to modify con-

\footnotetext{
${ }^{1}$ Radiative corrections to the $\chi^{+} \chi^{-}$production cross section are also available [16], but appear less crucial at present, as we discuss below.
} 
siderably the tree-level difference between the lightest chargino and neutralino masses, $m_{\chi^{ \pm}}-m_{\chi}$, which is one of the most important parameters controlling the efficiencies of experimental searches, especially in the "Higgsino" region of small $\mu$ and relatively large $M_{a}[17,18]$. More generally, the radiative corrections to the chargino and neutralino masses cause shifts in the inferred exclusion domains in the $\mu, M_{2}$ plane that are not negligible compared to those deduced from the improvements in the chargino mass limits obtained after successive LEP 2 energy upgrades. These changes are also comparable to the differences between mass limits obtained under different theoretical assumptions. Therefore, radiative corrections to the chargino and neutralino mass relations could in principle play a role in the delicate interplay of the direct experimental constraints used to constrain $m_{\chi}$ [11,5]. Clearly the degree of effort put into the experimental searches $[1-3]$ and their phenomenological interpretation merits the inclusion of these radiative corrections in the analysis of the LEP 2 data.

We find that there are significant shifts in the allowed regions of the $\mu, M_{2}$ plane. The effects on $m_{\chi^{ \pm}}$are particularly important when it is analyzed in terms of $M_{2}$, but not when it is analyzed as a function of $\Delta M \equiv m_{\chi^{ \pm}}-m_{\chi}$. We use these radiative corrections in an update of our previous analysis of LEP constraints in the MSSM parameter space, and on $m_{\chi}$ and $\tan \beta$ in particular, assuming universal input soft supersymmetry-breaking masses and a plausible cosmological relic density. This update also includes the most recent preliminary limits on chargino and neutralino production from LEP running at $183 \mathrm{GeV}$ [1-4], as well as new limits on the lightest neutral Higgs boson mass $m_{h}$ $[19,20,3,4]$. Relaxing the universal scalar mass assumption, we focus on the portion of the MSSM parameter space for which the lightest neutralino is a Higgsino, where the limits are particularly sensitive to the radiative corrections $[17,18]$. In this context, we evaluate the possibility of Higgsino dark matter, incorporating both coannihilations and radiative corrections in conjunction with the bounds imposed by LEP. We find only a very limited range of parameters in which Higgsino dark matter is viable, which will be explored completely by forthcoming LEP runs.

\section{IMPLEMENTATION OF RADIATIVE CORRECTIONS IN CHARGINO AND NEUTRALINO CODES FOR LEP}

As already mentioned, we work in the context of the MSSM in a supergravity framework where supersymmetry breaking is communicated to observable sparticles by gravity, so that the lightest neutralino $\chi$ is the lightest supersymmetric particle, and we also assume that $R$ parity is conserved, so that $\chi$ is stable. We further assume that the supersymmetry-breaking gaugino mass parameters are universal at the input supergravity scale: $M_{a}=m_{1 / 2}$. Similarly, we assume that the soft supersymmetry-breaking contributions to the squark and slepton masses $m_{\tilde{q}}, m_{\tilde{l}}$ are universal and equal to $m_{0}$ at the unification scale, and the trilinear parameters $A$ are also taken to be universal. The MSSM can then be parametrized by $m_{1 / 2}, m_{0}, A, \mu$, the pseudoscalar Higgs boson mass $m_{A}$, and $\tan \beta$. Here we project the experimental and cosmological bounds onto the $\mu, M_{2}$ plane for fixed $m_{0}, A, m_{A}$, and $\tan \beta$.

We note that only the trilinear coupling $A_{t}$, associated with the top-quark Yukawa coupling, is relevant for the phenomenology discussed here, ${ }^{2}$ and that the possible range of values of this parameter is constrained because of an infrared quasifixed point: $A_{t} \sim 2 m_{1 / 2} \sim 2 m_{\tilde{g}} / 3$ [21], so that this parameter is inessential. This quasifixed point arises because, if $A_{t}$ is much larger than $m_{1 / 2}$, the leading-order running of $A_{t}$ is given by $d A_{t} / d t \approx 6 \lambda_{t}^{2} A_{t} / 8 \pi^{2}$ [7], so that (for constant $\lambda_{t}$ ) $A_{t}$ decreases as a power law with scale, with an exponent that is roughly $1 / 12$ for $\lambda_{t} \sim 1$. Thus, if the input $A_{t} \gg m_{1 / 2}$, $A_{t}$ is reduced by an order of magnitude in its evolution from $M_{X}$ to $M_{Z}$. There are additional negative terms in the beta function for $A_{t}$ which are proportional to the gaugino masses, so that $m_{1 / 2}$ sets the scale for $A_{t}\left(M_{Z}\right)$. Consequently, $A_{t}\left(M_{Z}\right) \gg m_{1 / 2}$ requires extremely large $A_{t}\left(M_{X}\right)$. However, this tends to drive the stop soft mass-squared parameters negative at $M_{Z}$, so large values of $A_{t}\left(M_{Z}\right)$ are not allowed, at least for the values of $\tan \beta$ we consider. Therefore, we do not explore a range in values for $A_{t}$, but concentrate on the fixed-point value.

The input supersymmetry-breaking parameters are evolved down to the electroweak scale using the two-loop renormalization-group equations for the gaugino masses, so that the one-loop relation $M_{a}=\left(\alpha_{a} / \alpha_{\mathrm{GUT}}\right) \times m_{1 / 2}$ is violated at the $10 \%$ level. We also evolve the gauge and Yukawa couplings at the two-loop level, but other parameters are evolved with one-loop accuracy. At the tree level, we parametrize chargino and neutralino eigenstates in terms of $M_{2}, \mu$, and $\tan \beta$. We take a top mass of $171 \mathrm{GeV}$ : the only significant sensitivity of our results to $m_{t}$ comes in the Higgs mass constraints, which we discuss in Sec. V.

This theoretical framework has been adopted in many of the searches for supersymmetric particles at LEP, in particular for charginos and neutralinos. Until now, all the experimental analyses have used tree-level formulas for the masses and cross sections, in terms of the MSSM parameters introduced above. The one-loop calculations of the physical masses of the charginos and neutralinos [13-15], and more recently the $\chi^{+} \chi^{-}$production cross section [16], that have become available have been calculated in the dimensional reduction with modified minimal subtraction $(\overline{\mathrm{DR}})$ prescription, which is the supersymmetric analogue of the conventional modified minimal subtraction $(\overline{\mathrm{MS}})$ prescription. The one-loop mass formulas have now been incorporated [23] in one of the codes available for LEP experimental analyses, and we discuss here a first exploration of their implications for sparticle mass limits. The radiative corrections to the cross section are less important for this purpose: they are generally $\leqslant 15 \%$ or so [16], whereas the experimental upper limits on $\sigma_{+-} \equiv \sigma\left(e^{+} e^{-} \rightarrow \chi^{+} \chi^{-}\right)$are generally far below

\footnotetext{
${ }^{2}$ We do not consider very large values of $\tan \beta \gg 10$.
} 


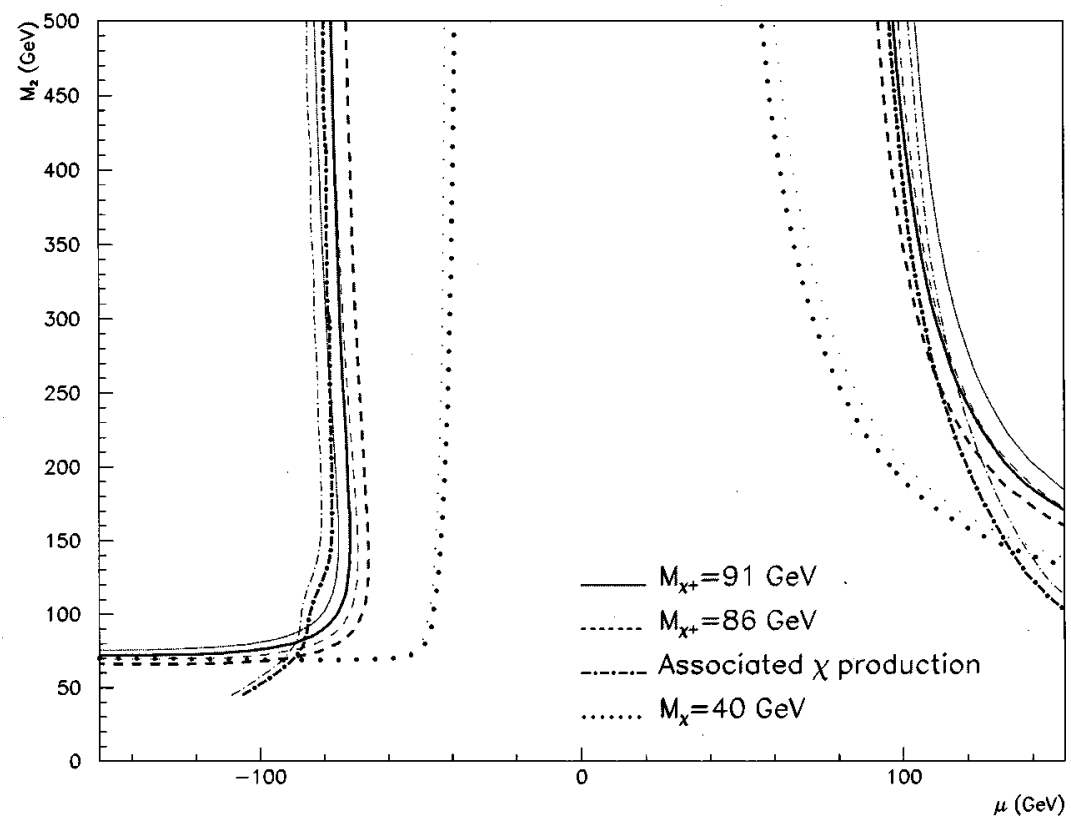

FIG. 1. The effects of one-loop radiative corrections in the $\mu, M_{2}$ plane, calculated for $\tan \beta$ $=2, \quad m_{0}=200 \mathrm{GeV}, \quad m_{A}=1 \mathrm{TeV}, \quad$ and $A_{t}$ $=2 m_{1 / 2}$. The thick lines are one-loop corrected contours corresponding to fixed values of chargino and neutralino masses, and the thin lines are tree-level contours. The continuous lines are for $m_{\chi^{ \pm}}=91 \mathrm{GeV}$, the dashed lines for $m_{\chi^{ \pm}}$ $=86 \mathrm{GeV}$, the dash-dotted lines for the bound on associated neutralino production, and the dotted lines for $m_{\chi}=40 \mathrm{GeV}$.

the theoretical predictions, except very close to the production threshold: $E_{\text {c.m. }} \sim 2 m_{\chi^{ \pm}}$. Since $\sigma_{+-}$varies very rapidly with $m_{\chi^{ \pm}}$in this region, the change the theoretical prediction for $\sigma_{+-}$has little effect on the chargino mass limit obtained.

\section{EXPLORATION OF CONSEQUENCES OF THE RADIATIVE CORRECTIONS}

Figure 1 displays the effects of these one-loop radiative corrections in the $\mu, M_{2}$ plane. The thick lines are one-loopcorrected contours corresponding to fixed values of chargino and neutralino masses, and the thin lines are tree-level contours. The continuous lines are for $m_{\chi^{ \pm}}=91 \mathrm{GeV}$, the dashed lines for $m_{\chi^{ \pm}}=86 \mathrm{GeV}$, corresponding, respectively, to the kinematic limits on $m_{\chi^{ \pm}}$from $e^{+} e^{-} \rightarrow \chi^{+} \chi^{-}$at the just completed $183 \mathrm{GeV}$ run of LEP and its previous run around $172 \mathrm{GeV}$. The dash-dotted lines show the contour of $m_{\chi}=40 \mathrm{GeV}$, corresponding to our previous lower limit on $m_{\chi}$ when several theoretical and cosmological constraints are applied [6].

In general, each of the four neutralinos $\chi_{i}$ is characterized by the four components $\alpha_{i}, \beta_{i}, \gamma_{i}$, and $\delta_{i}$ of its eigenvector in the $\widetilde{W}, \widetilde{B}, \widetilde{H}_{1}, \widetilde{H}_{2}$ basis. In this notation, the gaugino fraction of a particular neutralino $\chi_{i}$ is $\sqrt{\alpha_{i}^{2}+\beta_{i}^{2}}$, and its Higgsino fraction is $\sqrt{\gamma_{i}^{2}+\delta_{i}^{2}}$. In a later section of this paper, we discuss whether LEP limits still allow the lightest neutralino to be predominantly a Higgsino. With a view to this subsequent discussion, we recall here that the $Z^{0}$ coupling to a pair of neutralinos $\chi_{i}$ and $\chi_{j}$ is proportional to the Higgsino components of the neutralinos, namely, $\gamma_{i} \gamma_{j}-\delta_{i} \delta_{j}$. Thus it is particularly easy for LEP to probe regions of the $\mu, M_{2}$ plane where the lightest neutralino is mainly a Higgsino, by searching for production in association with a heavier neutralino state which is also mainly a Higgsino. Accordingly, we also plot in the relevant parts of Fig. 1 dotted lines representing the experimental limit of $0.18 \mathrm{pb}$ on associated neutralino production $e^{+} e^{-} \rightarrow \chi_{i} \chi_{j}: i+j>2$. In the region of interest for this analysis, this is dominated by $e^{+} e^{-}$ $\rightarrow \chi \chi_{2,3,4}$ and corresponds essentially to $m_{\chi}+m_{\chi_{H}^{\prime}}$ $=182 \mathrm{GeV}$, where $\chi_{H}^{\prime}$ is the lightest mainly Higgsino state among the $\chi_{2,3,4}$.

The one-loop radiative corrections in Fig. 1 are calculated with the following representative values of the input MSSM parameters: $\tan \beta=2, m_{0}=200 \mathrm{GeV}, m_{A}=1 \mathrm{TeV}$, and $A_{t}$ $=2 m_{1 / 2}$. We see significant shifts between the pairs of corresponding thick and thin lines, making manifest the significance of the one-loop radiative corrections. Varying $m_{0}$ between 100 and $1000 \mathrm{GeV}$ does not have a noticeable effect on the lines plotted, and they do not depend strongly on the precise value chosen for $m_{A}$, though they would be significantly different for values of $A_{t}$ much larger than the fixedpoint value that we favor. Interestingly, the differences between these pairs of lines are comparable to the splitting between the lines for $m_{\chi^{ \pm}}=86$ and $91 \mathrm{GeV}$, indicating that the radiative corrections have phenomenological implications for the inferred constraints on the input MSSM parameters $\mu, M_{2}$ that are comparable to the upgrade in the LEP energy from 172 to $183 \mathrm{GeV}$.

The LEP exclusions for charginos do not always extend to the kinematic limits. In particular, the lower limit on $m_{\chi^{ \pm}}$is sensitive to $m_{0}$ : it affects $\sigma_{+-}$through the $\tilde{\nu}$ exchange diagram, and when $m_{0}$ is such that $m_{\chi^{ \pm}}-m_{\tilde{\nu}}<3 \mathrm{GeV}$, chargino decays to a sneutrino and a low-energy lepton reduce the experimental efficiency to essentially zero. Moreover, as seen in Fig. 2, there is also a loss of sensitivity for fixed $m_{0}$ at large $M_{2}$, because of the reduction in detection efficiency that occurs when the difference in mass between the produced particle and its supersymmetric decay product, e.g., $\Delta M \equiv m_{\chi^{ \pm}}-m_{\chi}$, is small. This implies that at large $M_{2}$, beyond the top of Fig. 1, the experimental exclusion in the $\mu, M_{2}$ plane would contact toward $|\mu| \simeq 45 \mathrm{GeV}$. It has been observed previously $[17,18]$ that $\Delta M$ may be altered significantly by the one-loop radiative corrections, altering the 

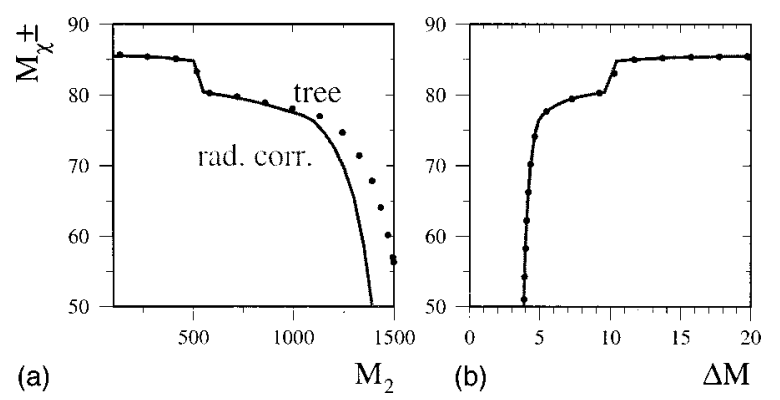

FIG. 2. (a) The experimental limit on $m_{\chi^{ \pm}}$as a function of $M_{2}$ and as a function of $\Delta M \equiv m_{\chi^{ \pm}}-m_{\chi}$ for fixed $m_{0}=200 \mathrm{GeV}$ and $\tan \beta=2$. The value of $\mu$ is determined by the combination $m_{\chi^{ \pm}}$and $M_{2}$. The drop at large $M_{2}$ is due to the loss in experimental efficiency as $\chi^{ \pm}$decays into $\chi$ involve softer leptons. The dotted line comes from a tree-level analysis, and the solid line is obtained using an ad hoc parametrization of the experimental efficiency, in conjunction with the radiatively corrected mass formulas. Note that the lines separate significantly when $M_{2}$ is large. (b) The experimental limit on $m_{\chi^{ \pm}}$is now plotted as a function of $\Delta M \equiv m_{\chi^{ \pm}}-m_{\chi}$. Note that the tree-level and radiatively corrected curves are almost coincident.

value of $M_{2}$ where this contraction would occur. For $\tan \beta$ not too large, the leading contribution to the change in $\Delta M$, $\delta(\Delta M)$, comes from heavy quark-squark loops, and for split top squark masses it is approximately given by $[17,18]$

$$
\delta(\Delta M)=\frac{3}{32 \pi^{2}} \lambda_{t}^{2} m_{t} \sin 2 \theta_{t} \ln \frac{\max \left(m_{t}^{2}, m_{\tilde{t}_{2}}^{2}\right)}{m_{\tilde{t}_{1}}^{2}},
$$

where $m_{t_{1}}>m_{t_{2}}$. Note that this vanishes in the limit that there is no stop mixing ( $\sin 2 \theta_{t} \rightarrow 0$ ), and that the sign of $\delta(\Delta M)$ depends on the sign of $\theta_{t}$. Both of these depend on the off-diagonal element of the stop mass-squared matrix, and hence on $A_{t}$. Hence, the quasifixed point for $A_{t}$ is an important constraint on radiative corrections to $\Delta M$.

Incorporating these radiative corrections fully in the analysis of experimental data would require the use in experimental simulations of the full radiative-correction code [23]. In the absence so far of such an analysis, we have used this code in conjunction with an ad hoc parametrization of the efficiency of the ALEPH chargino search [1] as a function of $\Delta M$ to explore the possible significance of this effect. We see in Fig. 2(a) that the exclusion contour in the $M_{2}, m_{\chi^{ \pm}}$ plane is indeed affected significantly. However, as seen in Fig. 2(b), this effect of the radiative corrections vanishes in the $\Delta M, m_{\chi^{ \pm}}$plane, as expected from the small change in the chargino composition. This suggests that a useful rule of thumb may be to expect that the exclusion domain in this particular plane may be insensitive to the radiative corrections also in a complete experimental analysis using Ref. [23].

\section{UPDATING LOWER LIMITS ON $m_{\chi}$ AND $\tan \beta$}

We now review the lower limit on $m_{\chi}$ derived previously [6] using data from the LEP runs at energies up to $172 \mathrm{GeV}$, discussing two issues: the significance of the radiative corrections and the impact of the more recent data at $183 \mathrm{GeV}$. The purely experimental constraint from LEP 172 coming from chargino production and associated neutralino production yielded the limit $m_{\chi}>14 \mathrm{GeV}$ [1], with the minimum occurring around $\tan \beta=3$. Adding the theoretical assumption that all squark masses are universal at the unification scale, unsuccessful Higgs searches indirectly strengthened the bound on $m_{\chi}$ significantly in the region around $\tan \beta$ $=2$ [6]. Furthermore, when scalar mass universality was extended to the Higgs sector and the values of $\mu$ and $m_{A}$ were fixed by requiring the consistency of electroweak symmetry breaking [22], the bound on $m_{\chi}$ was improved for all $\tan \beta$. For $\tan \beta \gtrsim 4$, we found $m_{\chi} \gtrsim 30 \mathrm{GeV}$, and the bound improved to $m_{\chi} \gtrsim 40 \mathrm{GeV}$ for $\tan \beta \lesssim 3$ [6].

We have also considered imposing the cosmological constraint that the neutralino relic density does not overclose the Universe. Specifically, the combination $\Omega_{\chi} h^{2}$ is constrained to be less than 0.3 by the requirement that the age of the Universe $t_{0}$ be greater than about $12 \mathrm{Gyr}$. These quantities are related through

$$
H_{0} t_{0}=\int_{0}^{1} d x(1-\Omega+\Omega / x)^{-1 / 2}
$$

where the scaled Hubble parameter is $h=H_{0} /$ $\left(100 \mathrm{~km} \mathrm{Mpc}^{-1} \mathrm{~s}^{-1}\right.$ ), and large relic densities may be incompatible with constraints on $t_{0}$. When the cosmological constraint was combined with the experimental constraints from Higgs boson searches and the theoretical assumption of universal Higgs boson masses, we found [6] that values of $\tan \beta \lesssim 1.7$ for $\mu<0$ and $\lesssim 1.4$ for $\mu>0$ were excluded. This is because the tree-level Higgs boson mass is small at low $\tan \beta$, so radiative corrections to $m_{h}$ must be enhanced by taking a large top squark mass. On the other hand, large sfermion masses lead to large relic densities for a gauginolike neutralino, as expected for universal scalar masses. Thus $\Omega_{\chi} h^{2}<0.3$ is not compatible with a heavy enough Higgs boson. Finally, when we imposed the astrophysical desire that the cosmological relic neutralinos play a significant role in structure formation, which requires that $\Omega_{\chi} h^{2}>0.1$, we found that the lower limit on the neutralino mass was strengthened at $\tan \beta \gtrsim 3$ to $m_{\chi} \geq 50 \mathrm{GeV}$.

In the interests of simplicity, we rediscuss here the lower bound only in the case in which all theoretical and cosmological assumptions are made, in particular that the input scalar masses are universal and that the relic cosmological density lies in the range $0.1 \leqslant \Omega_{\chi} h^{2} \leqslant 0.3$. The resulting bound on the lightest supersymmetric particle (LSP) mass in this case was previously found to be $m_{\chi} \geq 40 \mathrm{GeV}$, with the minimum being achieved in a narrow range of $2.6 \leqslant \tan \beta$ $\$ 3.0$, as seen in Fig. 1 of Ref. [6]. In this case, the lower bound on $m_{\chi}$ was linked directly to the lower limit $m_{\chi^{ \pm}}$ $\gtrsim 86 \mathrm{GeV}$ from the LEP $172 \mathrm{GeV}$ run and relied on the Higgs boson mass constraint. Cosmology did not play a direct role for this range of $\tan \beta$, though these constraints did play an important indirect role in closing off loopholes for special values of other MSSM parameters such as $\mu$ and $m_{0}$ where the limit on $m_{\chi^{ \pm}}$and hence $m_{\chi}$ could have been 
$\mathrm{M}_{\chi}=40 \mathrm{GeV}$ at tree level

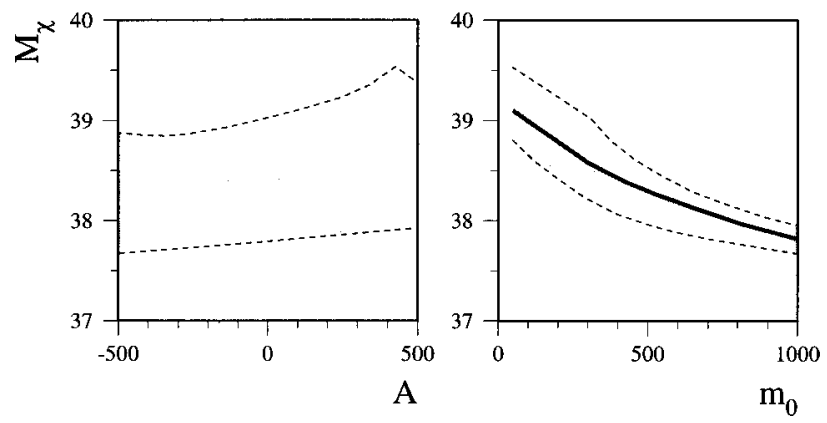

FIG. 3. Plots of the radiative correction to the lower limit on the neutralino mass, in the case that $m_{\chi^{ \pm}}=85 \mathrm{GeV} ; \tan \beta=2$ and $\mu$ $=-140 \mathrm{GeV}$. The plots are obtained by varying $-500 \mathrm{GeV} \leqslant A_{t}$ $\leqslant 500 \mathrm{GeV}$ and $50 \mathrm{GeV} \leqslant m_{0} \leqslant 1000 \mathrm{GeV}$. The solid line in the second panel is for the fixed-point value $A_{t}=2 m_{1 / 2}$. We see that the limit on $m_{\chi}$ is reduced by about $1 \mathrm{GeV}$ if we assume this value of $A_{t}$ and take $m_{0}=80 \mathrm{GeV}$, corresponding to the tree-level lower limit on $m_{\chi}$.

smaller, and provided stronger lower limits on $m_{\chi}$ for both lower and higher values of $\tan \beta$.

We turn first to the impact on this analysis of the radiative corrections discussed in the previous section. We display in Fig. 3 the radiatively corrected values of $m_{\chi}$ for $m_{\chi^{ \pm}}$ $=85 \mathrm{GeV}, \tan \beta=2$, and the representative value $\mu$ $=-140 \mathrm{GeV}$, corresponding to $m_{\chi}=40 \mathrm{GeV}$ at the tree level. The ranges plotted are generated by varying $A_{t}$ and $m_{0}$ over the ranges indicated in the two panels. We see that their effect is in general to reduce $m_{\chi}$ by $\sim 1$ to $2 \mathrm{GeV}{ }^{3}$ If we fix $A_{t}$ to the fixed-point value discussed earlier, we obtain the solid line shown in the second panel, and if we specify the value $m_{0} \sim 80 \mathrm{GeV}$ that corresponded to the minimum value of $m_{\chi}$ in our previous analysis, we find a reduction in $m_{\chi}$ by $\sim 1 \mathrm{GeV}$. We have investigated whether the roles of the LEP $172 \mathrm{GeV}$ Higgs boson limits and of cosmology are altered significantly when radiative corrections are included, and found that this is not the case. We conclude that the sensitivity of the previous LEP $172 \mathrm{GeV}$ lower limit on $m_{\chi}$ was around the $1 \mathrm{GeV}$ level.

We now turn to the implications of the LEP $183 \mathrm{GeV}$ data set. Again, for simplicity we restrict our attention to the case of universal soft supersymmetry-breaking scalar masses, including Higgs masses, implement the cosmological density constraint and focus on the region $\tan \beta \sim 2$ to 3 , where we previously found the lower bound on $m_{\chi}$ under these assumptions. The LEP lower limit on the chargino mass is now $m_{\chi^{ \pm}} \geqslant 91 \mathrm{GeV}$, corresponding to a strengthening of the lower limit on $m_{\chi}$ by $\sim 3 \mathrm{GeV}$. Including the shift downwards by $\sim 1 \mathrm{GeV}$ due to the radiative corrections, this direct LEP lower limit corresponds to $m_{\chi} \gtrsim 42 \mathrm{GeV}$. We have verified that the LEP Higgs boson searches and the cosmological density constraint continue to exclude loopholes in this limit.

The best limit on the Higgs boson of the standard model

\footnotetext{
${ }^{3}$ We have verified that this shift is numerically insensitive to $\mu$ over a range larger than that of relevance to this analysis.
}

is $88 \mathrm{GeV}$, which also applies to $m_{h}$ for $\tan \beta \lesssim 2$ in the MSSM $[19,20,3,4]$. The formulas we use to calculate $m_{h}$ are believed to have an uncertainty of about $2 \mathrm{GeV}$ [12], so we allow MSSM parameter sets for which they yield $m_{h}$ $\geqslant 86 \mathrm{GeV}$ at low $\tan \beta$. The LEP lower limit falls to about 78 $\mathrm{GeV}$ for $\tan \beta \gtrsim 3$, and we allow MSSM parameter sets for which $m_{h} \geqslant 76 \mathrm{GeV}$ for such larger values of $\tan \beta$. These Higgs boson limits strengthen the lower limit on $m_{\chi}$ for $\tan \beta \leqslant 2.6$ for $\mu<0$. Moreover, the strengthening of the LEP lower limit on $m_{\chi^{ \pm}}$, combined with cosmology, and including the two-loop running of the gaugino masses as well as the one-loop corrections to the chargino and neutralino masses, imply that $m_{\chi} \gtrsim 50 \mathrm{GeV}$ for $\tan \beta \geqslant 2.8$. Only in the region $2.6 \leqslant \tan \beta \leqslant 2.8$ for $\mu<0$ is there a narrow cleavage where $m_{\chi} \gtrsim 42 \mathrm{GeV}$ is still possible. There is no corresponding cleavage for $\mu>0$, where the previous lower bound $m_{\chi}$ $\gtrsim 50 \mathrm{GeV}$ for $\tan \beta \gtrsim 2$ remains essentially unchanged.

As we discussed above, for sufficiently low $\tan \beta$, one cannot satisfy simultaneously the Higgs mass constraint and the requirement that $t_{0}>12 \mathrm{Gyr}$. With the strengthening of the lower limit on $m_{h}$ from LEP 183, we now find that $\tan \beta \gtrsim 2.0$ for $\mu<0$ and $\tan \beta \gtrsim 1.65$ for $\mu>0$. Because the dependence of the Higgs mass on the top squark masses is only logarithmic, these bounds on $\tan \beta$ are quite insensitive to variations in $A_{t}$ or $t_{0}$. These strengthened results make it very difficult to reconcile our assumptions of universal scalar masses and an interesting cosmological relic density with the low $\tan \beta$ infrared fixed-point solution of the renormalization-group equations favored by some model builders [21].

\section{IMPLICATIONS FOR HIGGSINO DARK MATTER}

Although constrained supersymmetric models which impose universality on all the soft scalar masses, including the Higgs bosons, predict as a general feature that the lightest neutralino is a gaugino [24] as discussed above, it is well known that if the universality assumption is relaxed, the lightest supersymmetric particle can be a Higgsino over roughly half the parameter space as viewed on the $M_{2}, \mu$ plane [9], namely, when $|\mu| \lesssim M_{2}$, as seen in Fig. 4, which displays contours of higgsino and gaugino purities, as well as radiatively corrected $m_{\chi}$ contours. In this section we study whether it is still possible, in light of radiative corrections and the latest LEP data, for the lightest neutralino to be a Higgsino, even if one relaxes the scalar-mass-universality assumption.

For this purpose, our working definition of a Higgsino is that $p^{2}=\gamma_{i}^{2}+\delta_{i}^{2}>0.81$. In much of the Higgsino parameter space, it is possible to be even more definite. For small to moderate $M_{2}$, the lightest supersymmetric particle is the Higgsino combination defined by $\widetilde{S}^{0} \equiv \tilde{H}_{1}^{0} \cos \beta+\widetilde{H}_{2}^{0} \sin \beta$, i.e., $\gamma=\cos \beta$ and $\delta=\sin \beta$, with $m_{\tilde{S}_{0}} \rightarrow \mu \sin 2 \beta$ [9]. Contours of $\tilde{S}^{0}$ purity are displayed in Fig. 4 as dash-dotted lines. On the other hand, for large $M_{2}$, the lightest neutralino is the state $\widetilde{H}_{12} \equiv(1 / \sqrt{2})\left(\tilde{H}_{1}^{0} \pm \widetilde{H}_{2}^{0}\right)$, i.e., $\delta= \pm \gamma= \pm 1 / \sqrt{2}$ for $\operatorname{sgn}(\mu)= \pm 1$, with $m_{\tilde{H}_{12}} \rightarrow|\mu|$ [25]. Contours of $\tilde{H}_{12}$ purity 

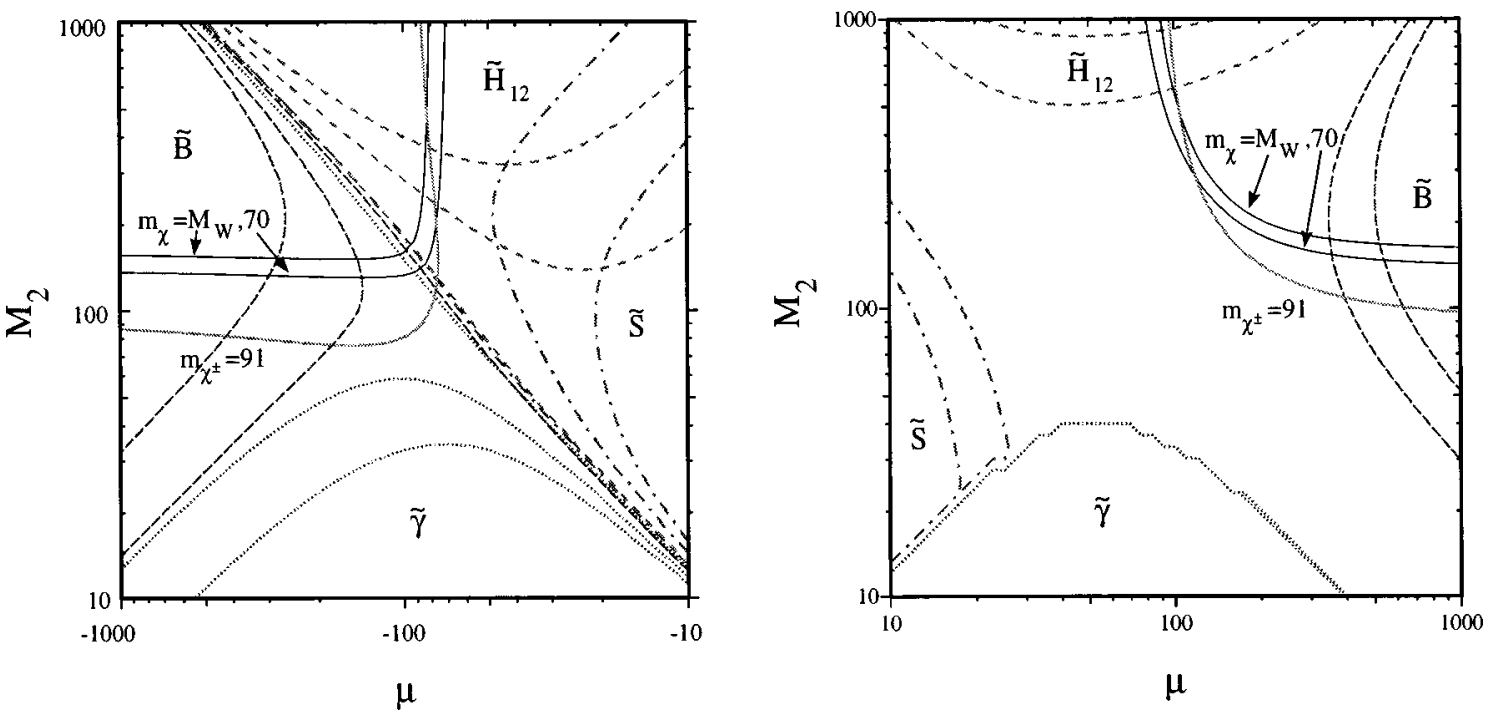

FIG. 4. Contours of neutralino purity: 99, 97, and (for $\mu<0$ ) $75 \%$, and chargino and neutralino masses (solid lines). The long-dashed lines are contours of high bino purity, the dotted lines are contours of high photino purity, the dashed lines are contours of high $\widetilde{H}_{12}$ Higgsino purity, and the dash-dotted lines are contours of high $\tilde{S}_{0}$ Higgsino purity. The radiative corrections to $m_{\chi}$ are calculated for tan $\beta=2$ and $m_{0}=100 \mathrm{GeV}$.

are shown as dashed lines in Fig. 4. The crossover point where the neutralino becomes more similar to $\widetilde{H}_{12}$ than $\widetilde{S}^{0}$ depends on $\mu$ and $\tan \beta$, but is typically at $M_{2}$ of a few hundred $\mathrm{GeV}[25]$, as seen in Fig. 4. The $\tilde{S}^{0}$ interacts much as a neutrino, i.e., it annihilates and scatters elastically via $Z$ exchange, with a coupling reduced by $\cos ^{2} 2 \beta$. On the other hand, a "pure", $\widetilde{H}_{12}$ state never annihilates or scatters through the $Z$, since the coupling $\gamma^{2}-\delta^{2} \rightarrow 0$. Because of its similarity to a heavy neutrino, the $\widetilde{S}^{0}$ may have an interesting relic density, with $\Omega_{\chi} h^{2}$ determined mainly by its mass and $\tan \beta$. However, this same property makes it ideal for exploration by LEP. For example, for $\tan \beta=2$, a $\tilde{S}^{0}$ state with $99 \%$ purity exists only for $|\mu|<25 \mathrm{GeV}$, and from Figs. 1 and 4 one can see that the region in the $\mu, M_{2}$ plane for such a pure state is explored by LEP, as discussed below in more detail. In contrast, the $\widetilde{H}_{12}$ has been very difficult to find or exclude. There are in principle two regions in the $\mu, M_{2}$ plane where $\widetilde{H}_{12}$ can provide an interesting relic density. For large $M_{2}$, they correspond to $|\mu|<M_{W}$ but with $m_{\chi^{ \pm}}$above the LEP limit, and $|\mu| \geqslant 1 \mathrm{TeV}$ [25]. The intermediate-mass Higgsino states are not of cosmological interest, because of their rapid annihilations to $W$ and $Z$ pairs. We have little to add concerning the very heavy $\widetilde{H}_{12}$ states, but the lighter Higgsinos lie directly in the region where the current LEP runs are eating away at the parameter plane, as we now discuss.

In the region where the lightest neutralino is $\tilde{H}_{12}$, this state is nearly degenerate with the associated chargino state, and in some cases with the second-lightest neutralino $\chi_{2}$. Therefore, computation of the relic abundance must then take into account the coannihilation with these degenerate partners [26,27]. The relic abundance is determined in this case by a Boltzmann equation in which the annihilation cross section is generalized to include coannihilation terms $\sigma_{\text {eff }}$ $=\sum \sigma_{i j} r_{i} r_{j}$, where $r_{i}$ is the relative abundance of particle $i$, and we include all three nearly degenerate states. The factors $r_{i}$ are in fact exponentially sensitive to the mass differences between the states. These mass differences are in turn sensitive to the radiative corrections to the neutralino and chargino masses, with potentially significant implications for the relic density, as pointed out in Ref. [18].

As a first approximation, the relic densities calculated with and without radiative corrections are rather similar for similar values of $\Delta M$. Deep in the Higgsino region at very large $M_{2}$, the mass difference between the LSP and chargino is very small, and coannihilations are very important in greatly reducing the relic density to uninteresting levels. In this limit, the small value of $\Delta M$ also makes this region inaccessible to LEP analysis. As one decreases $M_{2}$, the mass difference increases and co-annihilation becomes less important, thus increasing the relic density and the Higgsino's cosmological significance, while at the same time making this region readily accessible to LEP particle searches.

In Fig. 5 we display contours of constant chargino mass $m_{\chi^{ \pm}}=91 \mathrm{GeV}, m_{\chi}+m_{\chi_{H}^{\prime}}=182 \mathrm{GeV}$, Higgs mass $m_{h}$, and Higgsino purity, along with a contour of constant $\Omega_{\chi} h^{2}$ $=0.1$, for the choice $\tan \beta=2.0$ which is of interest for the subsequent discussion. Since the universal scalar mass constraint must be relaxed for Higgsino dark matter, we have chosen $m_{A}=m_{0}=1 \mathrm{TeV}$ so as to maximize the Higgs mass and hence minimize the impact of this constraint.

We see that the dashed lines in Fig. 5 representing the chargino and associated neutralino mass contours bound one away from small $|\mu|$, while the Higgs mass limit bounds one away from small $M_{2}$. This is particularly restrictive at low $\tan \beta$, where the tree-level Higgs mass is small, and thus where radiative corrections to $m_{h}$ must be enhanced by taking large top squark masses. The solid contour contains the 

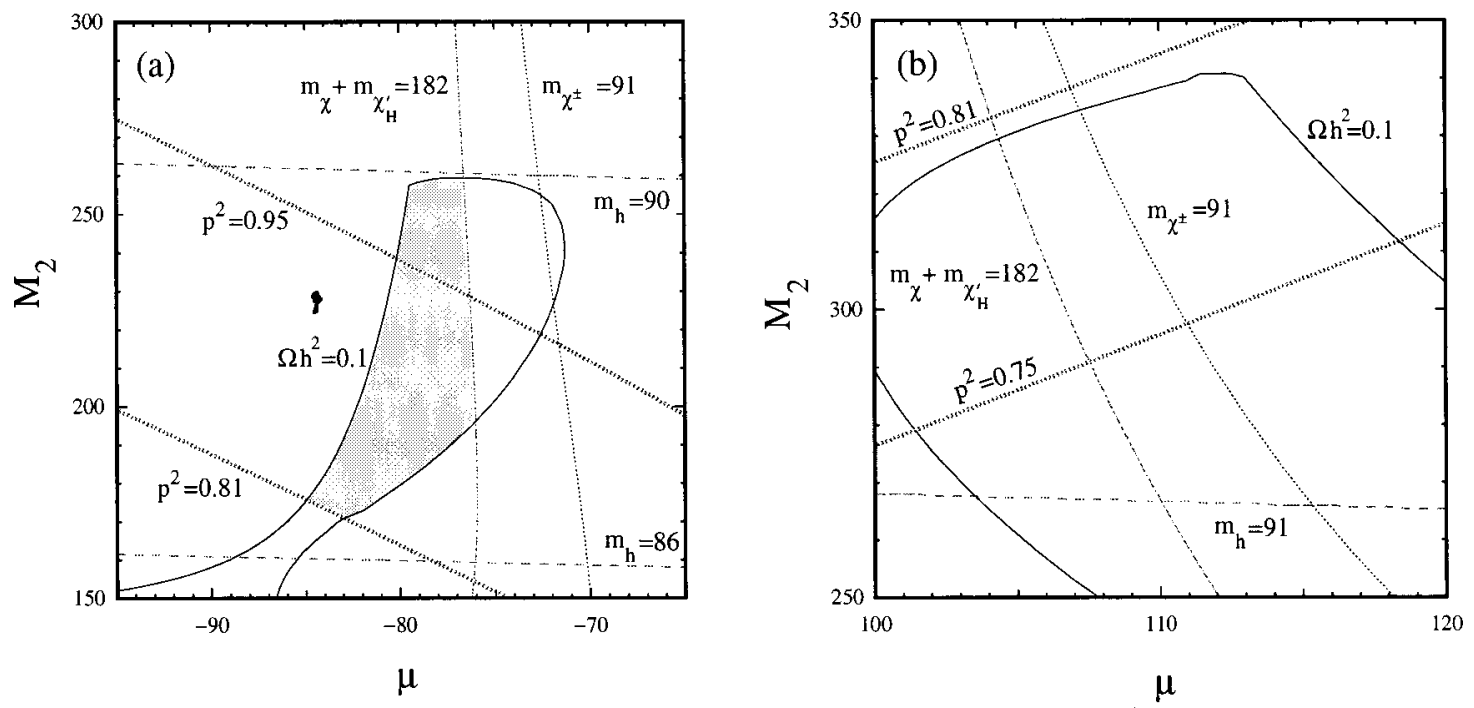

FIG. 5. Survey of experimental and cosmological constraints in the $\mu, M_{2}$ plane, focusing on Higgsino dark matter for tan $\beta=2$ and (a) $\mu<0$ and (b) $\mu>0$. We plot the radiatively corrected contours for $m_{\chi^{ \pm}}=91 \mathrm{GeV}$, for $m_{\chi}+m_{\chi_{H}^{\prime}}=182 \mathrm{GeV}$, for selected values of $m_{h}$ and the Higgsino purity $p$, and for $\Omega_{\chi} h^{2}=0.1$. The shaded regions yield a Higgsino which satisfies the mass and relic density constraints described in the text.

region which leads to a significant neutralino relic density $\Omega_{\chi} h^{2} \geqslant 0.1$, and its limited range in $M_{2}$ is a result of coannihilations. For larger values of $M_{2}$, the neutralino is a purer Higgsino, and the masses of both the lightest chargino and the next-to-lightest neutralino approach the neutralino mass from above, enhancing the effect of coannihilations that deplete the relic Higgsino abundance. For larger values of $|\mu|$, the relic density is suppressed by annihilations into $W$ pairs. The hashed contours in Fig. 5(a) represent Higgsino purities. Note the limited range of $\mu$ for which the mass and relic density constraints are satisfied. In Fig. 5(b) we show the equivalent plot for $\mu>0$; in this case the Higgsino purities are lower, and the entire dark matter region falls below the purity cutoff.

The combined effects of the above constraints, corresponding to the shaded regions of Fig. 5, are displayed for different values of $\tan \beta$ in Fig. 6. We have again taken $m_{0}$ $=m_{A}=1 \mathrm{TeV}$ to minimize the effects of the Higgs boson and chargino mass constraints. We find that there are no consistent Higgsino dark matter candidates for $\tan \beta \leqslant 1.8$ or $\geqslant 2.5$ for $\mu<0$, or for any value of $\tan \beta$ for $\mu>0$. The Higgs boson mass constraint cuts off the bottom of the allowed regions at low $\tan \beta$. When $\mu<0$ it becomes a relevant constraint for $\tan \beta<2.0$ and is responsible for the complete disappearance of the allowed region when $\tan \beta \leqslant 1.8$. Within the allowed regions displayed, the relic densities generally increase as $|\mu|$ is increased, until the neutralino mass, whose minimum value here is $\sim 71 \mathrm{GeV}$, becomes greater than $m_{W}$, at which point the $W^{+} W^{-}$annihilation channel opens, driving the relic $\Omega_{\chi} h^{2}$ below 0.1 . In any event, $\Omega_{\chi} h^{2}$ is never greater than 0.12 anywhere in the allowed regions for $\mu$ $<0$.

If one considers neutralinos which have a lower Higgsino purity, i.e., are more mixed states, then additional allowed regions appear. For example, if the purity condition is relaxed to $p^{2}>0.75$, then for $\mu>0$ there are regions of com- parable area to those in Fig. 6 for which $\Omega_{\chi} h^{2}>0.1$, and these regions extend over a range in $\tan \beta$ from 1.6 to about 25. Similarly, regions considerably smaller than those in Fig. 6 appear for $\mu<0$ at $\tan \beta>10$ and persist up to $\tan \beta$ of about 45 . At large $\tan \beta, A_{t}$ can vary somewhat from the quasifixed point, and as the Higgs boson mass is considerably larger than the current experimental bound, one has the freedom to play with $m_{0}$ to find regions of larger $\Omega_{\chi} h^{2}$. However, these regions remain very small, regardless of $A_{t}$ and $m_{0}$.

We have taken a large value of $m_{0}$, namely, $1 \mathrm{TeV}$, for which the large top squark masses produce large radiative

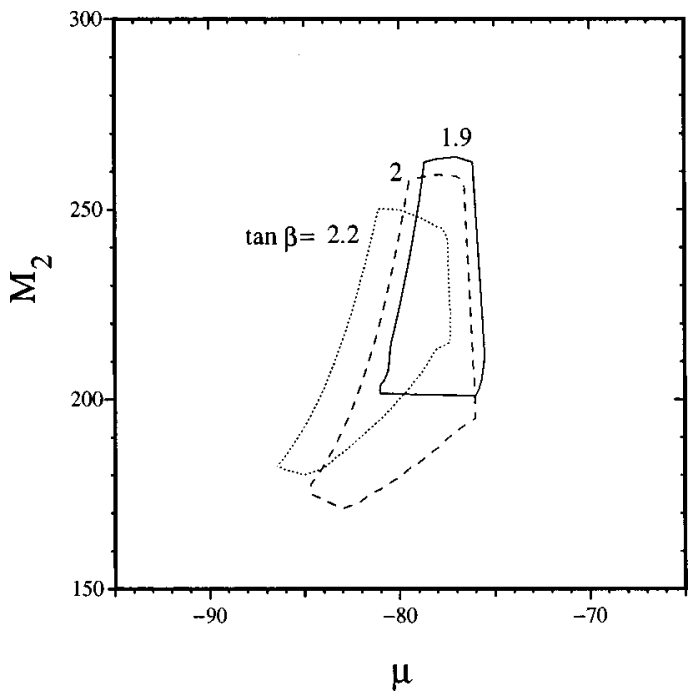

FIG. 6. The regions of the $\mu, M_{2}$ plane allowed by the constraints shown in the previous figure are shown for several different values of $\tan \beta$. There are no consistent choices of Higgsino parameters for $\tan \beta<1.8$ or $>2.5$ for $\mu<0$, or for any value of $\tan \beta$ for $\mu>0$. 

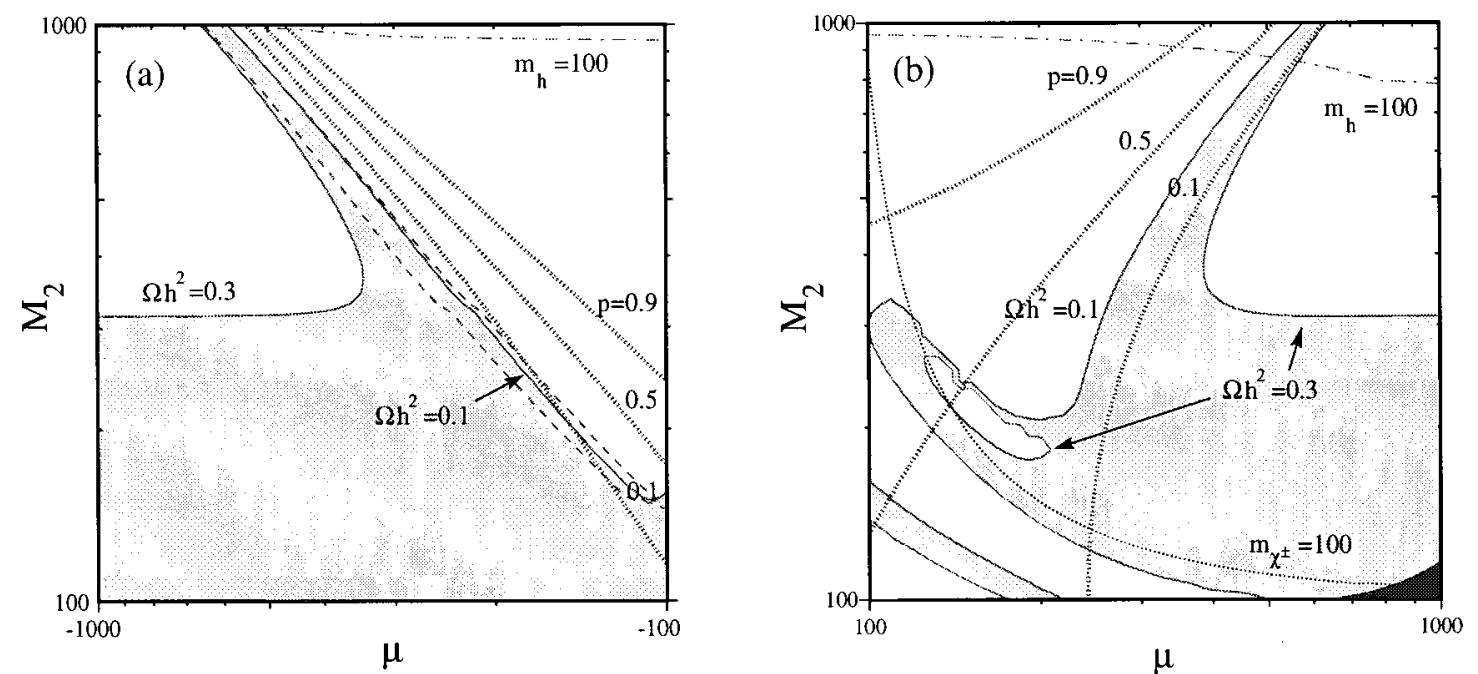

FIG. 7. Larger regions of the $\mu, M_{2}$ plane for $\tan \beta=2$ and (a) $\mu<0$, (b) $\mu>0$, with the radiatively corrected contours for $m_{\chi^{ \pm}}$ $=100 \mathrm{GeV}$ and $m_{h}=100 \mathrm{GeV}$, three choices of the Higgsino purity, and the $\Omega_{\chi} h^{2}=0.1,0.3$ contours for $m_{0}=100 \mathrm{GeV}$ and [in (a)] 1000 $\mathrm{GeV}$ (dashed lines). We see that the allowed regions for gaugino dark matter are very much larger than those for Higgsino dark matter shown in Fig. 5. The small dark shaded region in (b) yields a top squark with a negative mass squared.

corrections to $m_{h}$. On the other hand, we have chosen a value of $m_{t} 2 \mathrm{GeV}$ below the current central value, and a larger top quark mass would increase the radiative corrections. However, this is important only for the low values of $\tan \beta$ discussed above, where the Higgs mass constraint is important. On the other hand, if $\tan \beta$ is too small, a larger top quark mass will lead to a Landau pole in the evolution of $\lambda_{t}$. Because of this natural ceiling on the top quark mass as a function of $\tan \beta$, one cannot find any acceptable region for $\tan \beta<1.7$ for $\mu<0$, even pushing the top Yukawa quark to its perturbative maximum. For $\mu>0$, we have already taken $m_{t}$ as large as possible, hence the allowed range of $\tan \beta$ cannot be extended to lower $\tan \beta$ in this way.

Our allowed Higgsino regions shown in Figs. 5 and 6 are somewhat smaller than those depicted in Ref. [18], where the Higgsino region for negative $\mu$ extends up to $M_{2}$ $\sim 400 \mathrm{GeV}$ and down to $\mu \sim-60 \mathrm{GeV}$ for $\Omega h^{2}>0.1$. An important difference in our analysis is the strengthening of the LEP bounds, not only on the chargino mass (to about 91 $\mathrm{GeV}$ ), but on the associated production of Higgsinos (to $m_{\chi}+m_{\chi_{H}^{\prime}}>182 \mathrm{GeV}$, as seen in Fig. 5). This alone cuts out about $\frac{2}{3}$ of the Higgsino region of Ref. [18]. In addition, we have set the value of $A_{t}$ to its infrared quasifixed point $A_{t}$ $\sim 2 m_{1 / 2} \sim 2 m_{\tilde{g}} / 3$ [21] for the reasons discussed above, whereas a larger value $A_{t} \simeq 1 \mathrm{TeV}$ was chosen in Ref. [18]. Our choice of $A_{t}$ limits $a b$ initio the size of the radiative corrections which could remove the degeneracy between the Higgsino and chargino states. Thus, in our analysis the coannihilation of these particles remains important above $M_{2}$ $\gtrsim 250 \mathrm{GeV}$, in contrast to Ref. [18].

The corrections to the chargino and neutralino masses are positive in the regime studied, and also increase with the sfermion masses, although the corrections are much smaller than for $m_{h}$. Thus the chargino and associated neutralino production bounds tend to be more restrictive for lower $m_{0}$, unless one tunes $m_{\tilde{\nu}}$ so that it lies close to the chargino mass, in such a way that the production cross section is reduced by $\tilde{\nu}$ exchange and the experimental efficiency for the chargino search is reduced. This might weaken the chargino constraint, but the associated neutralino constraint is actually strengthened by positive interference from $\tilde{\nu}$ exchange. We also recall that the $\Omega_{\chi} h^{2}$ contours themselves are quite insensitive to the choice of $m_{0}$ in the deep Higgsino region, so there is no great net change in the boundaries of the allowed Higgsino regions in Figs. 5 and 6. On the other hand, as we discuss below, in regions where the gaugino admixture is greater than in Figs. 5 and 6, lowering $m_{0}$ decreases the relic density further as annihilations into fermions via sfermion exchange get enhanced. Last, as discussed above, the value of $A_{t}\left(m_{Z}\right)$ is quite insensitive to the choice of $A_{t} \cdot{ }^{4}$

The areas $\mathcal{A}$ of the allowed Higgsino regions are never very large: calculating them using the logarithmic measure $(d \mu / \mu)\left(d M_{2} / M_{2}\right)$, we find that their maxima are reached for $\tan \beta \sim 2$ for both $\mu<0$ and $\mu>0$, with $\mathcal{A}_{\max }=0.020$, 0.006 , respectively. Furthermore, the residual region for $\mu$ $<0$ could be explored by a search sensitive either to $m_{h}$ $\leqslant 100 \mathrm{GeV}$ or $m_{\chi}+m_{\chi_{H}^{\prime}} \leqslant 200 \mathrm{GeV}$, as anticipated if the LEP energy is eventually increased to $200 \mathrm{GeV}$, and the $\mu$ $>0$ region could be explored by a search for $m_{\chi^{ \pm}}$

\footnotetext{
${ }^{4} \mathrm{We}$ have checked what happens if we relax the gaugino mass unification constraint and take $M_{1}=M_{2}$ [28]. We find that the allowed regions are somewhat smaller than in the unified case for $\mu<0$ and disappear entirely for $\mu>0$. The latter results from a combination of larger neutralino masses-so that $m_{\chi}>m_{W}$ in a greater region, and closer $\chi^{ \pm}-\chi$ mass degeneracy-leading to a higher coannihilation rate.
} 
$\leqslant 100 \mathrm{GeV} .^{5}$ To put these numbers in perspective, one might wish to compare them with the logarithmic area of the full $\mu, M_{2}$ plane for values of these parameters between 100 and $1000 \mathrm{GeV}$, namely, $\mathcal{A} \sim 5$. Alternatively, one might wish to compare with the logarithmic area of the parts of this plane where gaugino dark matter is still viable. This can be visualized in Fig. 7, where we display the regions where 0.1 $\leqslant \Omega_{\chi} h^{2} \leqslant 0.3$ for $m_{0}=100 \mathrm{GeV}$ (solid contours) and $m_{0}$ $=1000 \mathrm{GeV}$ [dashed contours in Fig. 7(a)]. Here we choose $m_{A}=3000 \mathrm{GeV}$ to avoid the annihilation pole associated with the heavy Higgs boson, but the other parameter values are as in Fig. 5. In the region with high gaugino purity and low Higgsino content, annihilation is predominantly through sfermion exchange into fermion pairs. For $m_{0}=1000 \mathrm{GeV}$, annihilation through this channel is effectively shut off, leading to the very small allowed region demarcated by the dashed contours in Fig. 5(a). For $m_{0}=100 \mathrm{GeV}$, the cosmologically preferred region is significant. It is clear that the gaugino area is much larger than the remnant Higgsino area, indicating that this is a much more generic possibility. Indeed, the logarithmic area for gaugino dark matter is $\mathcal{A} \gtrsim 2$ for both $\mu$ positive and negative, some two orders of magnitude larger than the residual Higgsino regions.

\section{CONCLUSIONS}

The main purpose of this paper has been to include radiative corrections to chargino and neutralino masses for the first time in a phenomenological analysis of the LEP data. This analysis has been based on an implementation [23] of the analytical results published previously in one of the codes developed for LEP data analysis. This implementation makes it possible for radiative corrections to be taken into account in experimental analyses. In our first application of the radiatively corrected code [23], we have found that radiative corrections generically reduce the upper limit on the

\footnotetext{
${ }^{5} \mathrm{~A}$ fraction of the (very small) large $\tan \beta$ regions which open up for $\mu<0$ when the purity constraint is relaxed would not be probed by the LEP run at $200 \mathrm{GeV}$.
}

lightest neutralino mass by about $1 \mathrm{GeV}$ compared to what would be inferred from a tree-level analysis.

We have also updated our previous analysis of constraints from LEP and cosmology to include preliminary results from the latest LEP run at $183 \mathrm{GeV}$. Assuming scalar-mass universality, the previous lower limit on $m_{\chi}$ is now increased to about $42 \mathrm{GeV}$, and the previous lower bounds on $\tan \beta$ are increased to about 2.0 for $\mu<0$ and 1.65 for $\mu>0$, making life difficult for infrared fixed-point models.

We have also studied in detail the viability of Higgsino dark matter in light of the latest LEP constraints, relaxing the scalar-mass-universality assumption. The radiative corrections play an essential role, as do the LEP constraints on charginos, associated neutralino production, and Higgs bosons. We find that $m_{\chi} \gtrsim 71 \mathrm{GeV}$ in the remaining Higgsino region, that for $\mu<0$ there is only a very limited range of $\tan \beta$ over which Higgsino dark matter is viable at all, and that the area of the the $\mu, M_{2}$ plane within which it is viable is always very restricted. This is particularly evident when the Higgsino region is compared with the region of the $\mu, M_{2}$ plane over which gaugino dark matter is viable.

Finally, we review briefly the prospectives for further exploration of neutralino dark matter with higher-energy runs of LEP. We have already observed that prospective improvements in the sensitivity to MSSM Higgs bosons, charginos, and other supersymmetric particles should enable the complete investigation of neutralino dark matter up to $\sim 50 \mathrm{GeV}$, if universal input scalar masses and an interesting cosmological relic density are assumed. Additionally, the analysis of this paper indicates that the future higher-energy LEP runs should also provide closure on the possibility of Higgsino dark matter, by telling us whether the lighter chargino or the lightest MSSM Higgs boson weighs less than $100 \mathrm{GeV}$.

\section{ACKNOWLEDGMENTS}

This work was supported in part by U.S. DOE Grant No. DE-FG02-94ER-40823. The work of T.F. was supported in part by U.S. DOE Grant No. DE-FG02-95ER-40896 and in part by the University of Wisconsin Research Committee with funds granted by the Wisconsin Alumni Research Foundation.
[1] ALEPH Collaboration, R. Barate et al., Eur. Phys. J. C2, 417 (1998); DELPHI Collaboration, P. Abreu et al., ibid. C1, 1 (1998); L3 Collaboration, M. Acciarri et al., CERN Report No. PPE/97-130, 1997; OPAL Collaboration, K. Ackerstaff et al., Eur. Phys. J. C2, 213 (1998).

[2] Papers contributed to the International Europhysics Conference on High-Energy Physics, Jerusalem, August 1997 (http:// www.hep97.ac.il/hep97/abstract/papers.htm): ALEPH Collaboration, talk numbers 594, 614, 856: DELPHI Collaboration, talk numbers 348, 427, 858; L3 Collaboration, talk numbers 522, 859; OPAL Collaboration, talk numbers 209, 210.

[3] Presentations at the open LEPC session, Nov. 11th, 1997:
ALEPH Collaboration, P. Dornan, URL http:// alephwww.cern.ch/ALPUB/seminar/seminar.html; DELPHI Collaboration, P. Charpentier, URL http://wwwinfo.cern.ch/ $\sim$ charpent/LEPC/; L3 Collaboration, M. Pohl, URL http:// hpl3sn02.cern.ch/conferences/talks97.html; OPAL Collaboration, A. Honma, URL http://www.cern.ch/Opal

[4] For a recent experimental review, see P. Janot, Plenary talk at the International Europhysics Conference on High-Energy Physics, Jerusalem, August, 1997 (unpublished).

[5] J. Ellis, T. Falk, K. A. Olive, and M. Schmitt, Phys. Lett. B 388, 97 (1996).

[6] J. Ellis, T. Falk, K. A. Olive, and M. Schmitt, Phys. Lett. B 413, 355 (1997). 
[7] For reviews, see H. P. Nilles, Phys. Rep. 110, 1 (1984); H. E. Haber and G. L. Kane, ibid. 117, 75 (1995).

[8] H. Goldberg, Phys. Rev. Lett. 50, 1419 (1983).

[9] J. Ellis, J. S. Hagelin, D. V. Nanopoulos, K. A. Olive, and M. Srednicki, Nucl. Phys. B238, 453 (1984).

[10] J. Ellis, in Topics in Electroweak Physics, Proceedings of the 1996 Lake Louise Winter Institute, edited by A. Astbury, B. A. Campbell, F. C. Khanna, and J. L. Pinfold (World Scientific, Singapore, 1997), p. 40; K. A. Olive, astro-ph/9707212.

[11] ALEPH Collaboration, D. Buskulic et al., Z. Phys. C 72, 549 (1996).

[12] H. E. Haber, R. Hempfling, and A. H. Hoang, Z. Phys. C 75 539 (1997); see also M. Carena, M. Quiros, and C. E. M. Wagner, Nucl. Phys. B461, 407 (1996).

[13] A. B. Lahanas, K. Tamvakis, and N. D. Tracas, Phys. Lett. B 324, 387 (1994).

[14] D. Pierce and A. Papadopoulos, Phys. Rev. D 50, 565 (1994); Nucl. Phys. B430, 278 (1994).

[15] D. M. Pierce, J. A. Bagger, K. Matchev, and R.-J. Zhang, Nucl. Phys. B491, 3 (1997).

[16] M. A. Diaz, S. F. King, and D. A. Ross, CERN Report No. TH/97-313, hep-ph/9711307; hep-ph/9801373.

[17] G. F. Giudice and A. Pomarol, Phys. Lett. B 372, 253 (1996).

[18] M. Drees, M. M. Nojiri, D. P. Roy, and Y. Yamada, Phys. Rev. D 56, 276 (1997).

[19] ALEPH Collaboration, R. Barate et al., Phys. Lett. B 412, 173 (1997); Report No. PPE/97-072 1997; DELPHI Collaboration, P. Abreu et al., Eur. Phys. J. C2, 1 (1998); L3 Collaboration, M. Acciarri et al., Phys. Lett. B 411, 373 (1997); OPAL Col- laboration, K. Ackerstaff et al., Eur. Phys. J. C1, 425 (1998).

[20] Papers contributed to the International Europhysics Conference on High-Energy Physics, Jerusalem, August 1997 (http:// www.hep97.ac.il/hep97/abstract/papers.htm): ALEPH Collaboration, talk numbers 748, 749; DELPHI Collaboration, talk numbers 425, 529; L3 Collaboration, talk numbers 518, 519; OPAL Collaboration, talk numbers 206, 207.

[21] M. Carena, M. Olechowski, S. Pokorski, and C. E. M. Wagner, Nucl. Phys. B426, 269 (1994), and references therein.

[22] For a review and references, see J. Ellis, in Proceedings of the 17th International Symposium on Lepton-Photon Interactions, Beijing 1995, edited by Z.-P. Zheng and H.-S. Chen (World Scientific, Singapore, 1996), p. 170.

[23] Routines to calculate the one-loop radiative corrections may be obtained from T. F. at falk@ pheno.physics.wisc.edu A package for MSSM calculations including these one-loop routines may be obtained from G. G. at Gerardo.Ganis@cern.ch

[24] J. Ellis, J. Hagelin, and D. V. Nanopoulos, Phys. Lett. 159B, 26 (1985); M. M. Nojiri, Phys. Lett. B 261, 76 (1991); J. Lopez, D. V. Nanopoulos, and K. Yuan, ibid. 267, 219 (1991); Nucl. Phys. B370, 445 (1992).

[25] K. A. Olive and M. Srednicki, Phys. Lett. B 230, 78 (1989); Nucl. Phys. B355, 208 (1991); K. Griest, M. Kamionkowski, and M. S. Turner, Phys. Rev. D 41, 3565 (1990).

[26] K. Griest and D. Seckel, Phys. Rev. D 43, 3191 (1991).

[27] S. Mizuta and M. Yamaguchi, Phys. Lett. B 298, 120 (1993).

[28] G. L. Kane and James D. Wells, Phys. Rev. Lett. 76, 4458 (1996). 